\title{
Finite Analytic Method for One-Dimensional Nonlinear Consolidation under Time-Dependent Loading
}

\author{
Dawei Cheng, ${ }^{1,2}$ Wenke Wang, ${ }^{1,2}$ Xi Chen, ${ }^{3}$ and Zaiyong Zhang ${ }^{1,2}$ \\ ${ }^{1}$ School of Environmental Science and Engineering, Chang'an University, Xian, China \\ ${ }^{2}$ Key Laboratory of Subsurface Hydrology and Ecological Effect in Arid Region, Chang'an University, \\ Ministry of Education, China \\ ${ }^{3}$ School of Civil Engineering and Architecture, Shaanxi Sci-Tech University, Hanzhong, China
}

Correspondence should be addressed to Dawei Cheng; cheng198493@qq.com

Received 23 October 2016; Accepted 2 March 2017; Published 30 March 2017

Academic Editor: Longjun Dong

Copyright (C) 2017 Dawei Cheng et al. This is an open access article distributed under the Creative Commons Attribution License, which permits unrestricted use, distribution, and reproduction in any medium, provided the original work is properly cited.

For one-dimensional (1D) nonlinear consolidation, the governing partial differential equation is nonlinear. This paper develops the finite analytic method (FAM) to simulate 1D nonlinear consolidation under different time-dependent loading and initial conditions. To achieve this, the assumption of constant initial effective stress is not considered and the governing partial differential equation is transformed into the diffusion equation. Then, the finite analytic implicit scheme is established. The convergence and stability of finite analytic numerical scheme are proven by a rigorous mathematical analysis. In addition, the paper obtains three corrected semianalytical solutions undergoing suddenly imposed constant loading, single ramp loading, and trapezoidal cyclic loading, respectively. Comparisons of the results of FAM with the three semianalytical solutions and the result of FDM, respectively, show that the FAM can obtain stable and accurate numerical solutions and ensure the convergence of spatial discretization for 1D nonlinear consolidation.

\section{Introduction}

In geotechnical engineering, subsoil is subjected to complicated time-dependent loading paths which are induced by different vibration sources, such as wave action, groundwater level cyclic variation, filling and discharging in silos and tanks, vehicular traffic, blast, and earthquake. In these vibration sources, such as waves, changes in water table, loading and unloading in the granary and water towers, and vehicle traffic can cause regular time-dependent loading paths $[1,2]$. However, time-dependent loading paths induced by blast, earthquake, and so on are irregular $[1,3]$. Many discriminant methods, just like the waveform spectrum analysis, multivariate statistical methods, and soft computing techniques, can identify these vibration sources $[4,5]$.

Based on the cyclic loading classification discriminant criteria proposed by Zienkiewicz and Bettess [6], these relatively regular time-dependent loading paths, induced by wave action, groundwater level cyclic variation, and so forth, are considered as low frequency cyclic loadings. Three effects, including the soil movement inertia force and relative movement inertia force between pore water and soil skeleton, can be ignored in soil consolidation behavior under low frequency loadings. Many methods can be used to simulate these low frequency cyclic loadings, such as suddenly imposed constant loading, single ramp loading, rectangular cyclic loading, triangle cyclic loading, and trapezoidal cyclic loading [7-9]. A period of trapezoidal cyclic loadings includes four phases: loading phase, the maximum load phase, unloading phase, and rest phase, respectively. It is well known that when the duration time of the maximum loading phase is 0 , the trapezoidal cyclic loading will be transformed into the triangle cyclic loading. However, when the duration time of loading and unloading phase is 0 , the trapezoidal cyclic loading will be transformed into the rectangular cyclic loading [10]. Therefore, this paper will discuss the trapezoidal cyclic loading as a result of its flexibility. In addition, the suddenly imposed constant loading is a basic load form and the single ramp loading is the simplest time-dependent loading path. Thus, both of them are also discussed. 
The problem of consolidation under time-dependent loading has received attention by various authors [11-13]. Based on the linear relationship of the soil's constitutive relation, Terzaghi and Fröhlich [11] extended Terzaghi's linear consolidation theory to various cases of time-dependent loading following a single ramp loading. Olson [12] presented charts for 1D consolidation for the case of simple ramp loading assuming a constant consolidation coefficient. Favaretti and Soranzo [13] derived solutions for common types of cyclic loadings. However, soil's constitutive relationships are actually nonlinear $[10,14]$. The coefficient of compressibility decreases with increasing effective stress. In addition, the permeability coefficient decreases with void ratio decrease.

Davis and Raymond [14] developed a nonlinear theory of consolidation assuming the relationship between void ratio and the logarithm of effective stress to conform to linear law, and the decrease in permeability coefficient is proportional to the compressibility. Xie et al. [10] derived a semianalytical solution for trapezoidal cyclic loading by assuming the validity of Davis's nonlinear theory of consolidation. Razouki et al. [15] derived an analytical solution for consolidation under haversine cyclic loading based on the same assumption. Geng et al. [7] developed a semianalytical method to solve $1 \mathrm{D}$ consolidation behavior taking into account the relationship between void ratio and the logarithm of linear effective stress responses. Research has revealed that a stress-strain relation curve is more aligned with the hyperbolic model for certain types of soil, such as soft clay $[16,17]$. Shi et al. [18] derived a semianalytical solution for consolidation under suddenly imposed constant loading, taking into account the stress-strain relation curve with a hyperbolic model and the decrease in permeability coefficient being proportional to the decrease in compressibility. Zhang et al. [19] adopted the same assumption, deriving a semianalytical solution for consolidation under trapezoidal cyclic loading. But, for simplifying nonlinear consolidation model, the assumptions of constant initial effective stress are often used, such as Davis's model and Zhang's model, which does not conform to practical condition and limits the application of those models in various initial conditions.

It is worth noting that the governing partial differential equation is nonlinear partial differential equation. It is difficult to obtain analytical solution, except under specific conditions. So developing numerical solutions for solving complex realistic problems is necessary. As a methodology, the finite analytic method (FAM) was first introduced to mainly solve heat conduction and Navier-Strokes equations [20, 21]. The combination, under FAM of the numerical method and analytical method, gives higher precision, good numerical stability, and fast convergence and is widely employed in fluid mechanics and groundwater dynamics [22-25].

In this paper, FAM is first developed to solve the governing partial differential equation of $1 \mathrm{D}$ nonlinear consolidation, taking into account the stress-strain behavior expressed by a hyperbolic model and where the permeability coefficient is proportional to compressibility. Then three corrected semianalytical solutions undergoing suddenly imposed constant loading, single ramp loading, and trapezoidal cyclic loading are, respectively, obtained, without the assumption of constant initial effective stress. Finally, the numerical solution of FAM is compared with these three semianalytical solutions and the numerical solution of finite difference method (FDM), respectively.

\section{The Governing Partial Differential Equation of 1D Nonlinear Consolidation}

Modify Terzaghi's hypothesis as follows [19]:

(a) According to results of oedometer tests, the stressstrain relation curve is fitted with a hyperbolic model $[16,17]$ :

$$
\frac{\sigma^{\prime}}{\varepsilon}=E+n \sigma^{\prime}
$$

where $\sigma^{\prime}$ is the effective stress; $\varepsilon$ is the vertical strain; $E$ is the initial elastic modulus; $n=1 / \varepsilon_{f}$ is the reciprocal of the final vertical strain. When $n$ equals zero, the stress-strain relationship is linear.

(b) According to results of oedometer tests, the coefficient of consolidation $C_{v}$ varies much less than the compressibility coefficient $\alpha_{v}$ and may be taken as constant; that is, the decrease in permeability coefficient $k$ is proportional to the decrease in compressibility coefficient $\alpha_{v}[14]$. The coefficient of consolidation $C_{v}$ is constant; that is,

$$
C_{v}=\frac{k\left(1+e_{0}\right)}{\alpha_{v}}=\text { const }
$$

where $e_{0}$ is the initial void ratio.

(c) The initial effective stress is constant; that is,

$$
\sigma_{0}^{\prime}=\frac{\int_{0}^{L} \gamma^{\prime} z d z}{L}=\frac{1}{2} \gamma^{\prime} L,
$$

where $\gamma^{\prime}$ is the soil effective gravity and $L$ is the depth of the calculation.

(d) Imposed loadings change with time.

Other assumptions are the same as those in Terzaghi's theory.

Based on the abovementioned assumptions, except hypothesis (c), the governing partial differential equation of $1 \mathrm{D}$ consolidation for time-dependent loading [19] can be established as follows:

$$
\begin{aligned}
& -C_{v} E\left[\frac{1}{\left(E+n \sigma^{\prime}\right)^{2}} \frac{\partial^{2} u}{\partial z^{2}}-\frac{2 n}{\left(E+n \sigma^{\prime}\right)^{3}} \frac{\partial \sigma^{\prime}}{\partial z} \frac{\partial u}{\partial z}\right] \\
& =\frac{E}{\left(E+n \sigma^{\prime}\right)^{2}} \frac{\partial \sigma^{\prime}}{\partial t},
\end{aligned}
$$

where $u$ is excess pore-water pressure and $z$ is the height beneath the upper boundary.

Note that hypothesis (c) means that the initial effective stress is the same for every point in depth, which does not conform to practical condition and limits the application of (4) in various initial conditions. Thus, hypothesis (c) will not be considered in the paper. 


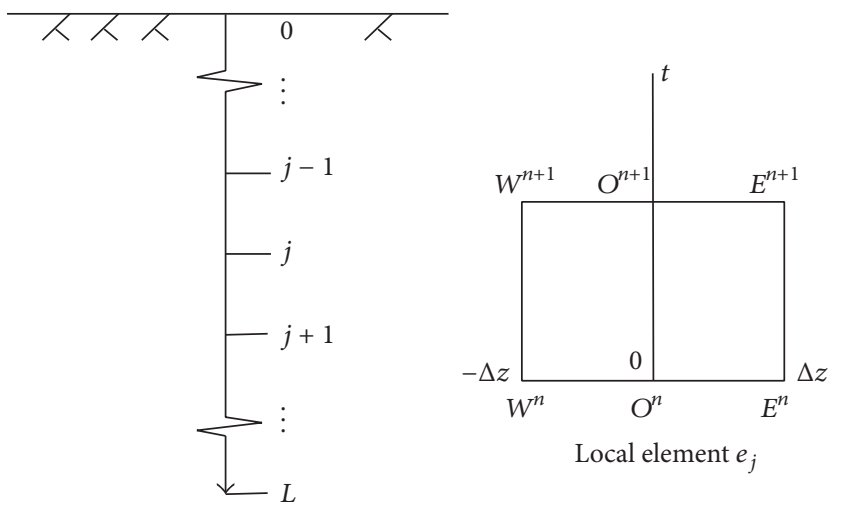

FIgURE 1: Domain and local element of finite analytical method.

By the principle of effective stress, this becomes

$$
\sigma^{\prime}=q(t)-u
$$

where $q(t)$ is cyclic loading.

By defining a new parameter $\omega$,

$$
\omega=\frac{\left(1+e_{0}\right) \sigma^{\prime}}{E+n \sigma^{\prime}}-\frac{\left(1+e_{0}\right) q(t)}{E+n q(t)},
$$

(4) can be simplified to the following form:

$$
C_{v} \frac{\partial^{2} \omega}{\partial z^{2}}=\frac{\partial \omega}{\partial t}+R(t)
$$

where $R(t)=\left(E\left(1+e_{0}\right) /[E+n q(t)]^{2}\right)(d q(t) / d t)$.

\section{Finite Analytic Scheme}

In the FAM, the modeling domain of (7) can be discretized into small elements by using the spatial discretization $\Delta z$ (Figure 1). A typical FAM element $e_{j}$ with the space step $2 \Delta z$ in a given time interval $\Delta t=t^{n}-t^{n-1}$ is shown in Figure 1. The origin of local coordinates is symbolized by $O$. Therefore, the local element is

$$
e_{j}=\{-\Delta z \leq z \leq \Delta z, 0 \leq t \leq \Delta t\}
$$

The space term is treated as the implicit scheme of the time term in local element $e_{j}$. The unsteady term $\partial \omega / \partial t$ takes the values at point $O$ at the $n$th time step. $R(t)$ also takes the values at the $n$th time step. At the $(n+1)$ th time step, (7) can be written as follows:

$$
C_{v}\left(\frac{\partial^{2} \omega}{\partial z^{2}}\right)^{n+1}=\left.\frac{\partial \omega}{\partial t}\right|_{O} ^{n}+\left.R(t)\right|^{n}
$$

where $\left(\partial^{2} \omega / \partial z^{2}\right)^{n+1}$ indicates the values of the element at $(n+$ 1)th time step; $\left.(\partial \omega / \partial t)\right|_{O} ^{n}$ indicates the unsteady term taking the values at point $O$ at the $n$th time step; $\left.R(t)\right|^{n}$ indicates that $R(t)$ takes the values at the $n$th time step.
The unsteady term $\partial \omega / \partial t$ is approximated by $\left(\omega_{O}^{n+1}-\right.$ $\left.\omega_{O}^{n}\right) / \Delta t$, and (9) can be normalized as follows:

$$
\left(\frac{\partial^{2} \omega}{\partial z^{2}}\right)^{n+1} \approx \frac{1}{C_{v}}\left(\frac{\omega_{O}^{n+1}-\omega_{O}^{n}}{\Delta t}+\left.R(t)\right|_{t=n \Delta t}\right),
$$

where $\omega_{O}^{n+1}$ and $\omega_{O}^{n}$ indicate the unsteady term taking the values at point $O$ at the $(n+1)$ th and $n$th time step, respectively; $\left.R(t)\right|_{t=n \Delta t}$ indicates that $R(t)$ takes the values at the $n$th time step.

Here,

$$
f=\frac{1}{C_{v}}\left\{\frac{\omega_{O}^{n+1}-\omega_{O}^{n}}{\Delta t}+R(n \Delta t)\right\} .
$$

At the $(n+1)$ th time step, (11) can be written as follows:

$$
\left(\frac{\partial^{2} \omega}{\partial z^{2}}\right)^{n+1}=f
$$

The general solution of (12) is

$$
\omega^{n+1}(z)=f \frac{z^{2}}{2}+c_{1} z+c_{2}
$$

where the constants $c_{1}$ and $c_{2}$ can be specified by two nodal values of $\omega$ at the $n$th time step ( $W^{n}$ and $E^{n}$ in Figure 1); that is,

$$
\begin{aligned}
& \omega_{W}=f \frac{\Delta z^{2}}{2}-c_{1} \Delta z+c_{2} \quad z=-\Delta z, \\
& \omega_{E}=f \frac{\Delta z^{2}}{2}+c_{1} \Delta z+c_{2} \quad z=\Delta z .
\end{aligned}
$$

Solving a system of equations composed of (14), the constants $c_{1}$ and $c_{2}$ are

$$
\begin{aligned}
& c_{1}=\frac{\omega_{E}-\omega_{W}}{2 \Delta z}, \\
& c_{2}=\frac{\omega_{E}+\omega_{W}}{2}-\frac{f}{2} \Delta z^{2} .
\end{aligned}
$$

Substituting (15) into (13) and letting the value of $z$ be zero,

$$
\omega_{O}^{n+1}=\frac{\omega_{E}+\omega_{W}}{2}-\frac{f}{2} \Delta z^{2}
$$

Substituting (11) into (16),

$$
\begin{aligned}
\omega_{O}^{n+1}= & \frac{1}{2+\Delta z^{2} / C_{v} \Delta t} \omega_{E}+\frac{1}{2+\Delta z^{2} / C_{v} \Delta t} \omega_{W} \\
& +\left[\frac{\Delta z^{2} / C_{v} \Delta t}{2+\Delta z^{2} / C_{v} \Delta t}\right] \omega_{O}^{n} \\
& -\frac{\Delta z^{2} / C_{v}}{2+\Delta z^{2} / C_{v} \Delta t} R(n \Delta t) .
\end{aligned}
$$


Supposing $\lambda=\Delta z^{2} / C_{v} \Delta t$, the local analytical solution for the element $e_{j}$ is then written as follows:

$$
\begin{aligned}
\omega_{O}^{n+1}= & \frac{1}{2+\lambda} \omega_{E}+\frac{1}{2+\lambda} \omega_{W}+\left(\frac{\lambda}{2+\lambda}\right) \omega_{O}^{n} \\
& -\frac{\lambda \Delta t}{2+\lambda} R(n \Delta t) .
\end{aligned}
$$

This local analytical solution evaluated at the interior node $j$ of the element $e_{j}$ at the $n$th time step is

$$
\begin{aligned}
\omega_{j}^{n+1}= & \frac{1}{2+\lambda} \omega_{j-1}^{n+1}+\frac{1}{2+\lambda} \omega_{j+1}^{n+1}+\left(\frac{\lambda}{2+\lambda}\right) \omega_{j}^{n} \\
& -\frac{\lambda \Delta t}{2+\lambda} R(n \Delta t) .
\end{aligned}
$$

Equation (19) is the FAM implicit scheme of the governing partial differential equation of $1 \mathrm{D}$ nonlinear consolidation. A set of algebraic equations can be formed from (19), which can be solved by the method of forward elimination and backward substitution. Associated with boundary conditions, the algebraic equations are solved iteratively for the variable $\omega_{j}^{n}(n=1,2,3, \ldots, N, j=1,2,3, \ldots, M)$. Here, $N$ is the number of total time steps, and $M$ is the number of total nodes in the domain. The excess pore-water at the node $j$ and the $n$th time step is obtained by applying an inversion transformation for (5) and (6).

\section{The Convergence and Stability of the FAM Scheme}

4.1. Convergence of Finite Analytical Numerical Scheme. Errors of the FAM implicit scheme (19) are found by using (10). To demonstrate the convergence of FAM, the following equation is used to replace $\partial \omega / \partial t$ in (9):

$$
\left.\frac{\partial \omega}{\partial t}\right|_{j} ^{n}=\frac{\omega_{j}^{n}-\omega_{j}^{n-1}}{\Delta t}+O(\Delta t)
$$

Here, $O(\Delta t)$ is truncation error. $\omega_{j}^{n}$ represents the exact solution and $\widetilde{\omega}_{j}^{n}$ represents finite analytic numerical solution at the node $j$ and the $n$th time step, respectively. The error between the exact solution $\omega_{j}^{n}$ and finite analytic numerical solution $\widetilde{\omega}_{j}^{n}$ can be written as $\varepsilon_{j}^{n}$; thus,

$$
\varepsilon_{j}^{n}=\omega_{j}^{n}-\widetilde{\omega}_{j}^{n}
$$

$\omega_{j}^{n}$ should satisfy the following:

$$
\begin{aligned}
\omega_{j}^{n}= & \frac{1}{2+\lambda} \omega_{j-1}^{n}+\frac{1}{2+\lambda} \omega_{j+1}^{n}+\left(\frac{\lambda}{2+\lambda}\right) \omega_{j}^{n-1} \\
& -\frac{\lambda \Delta t}{2+\lambda} R(n \Delta t)+O(\Delta t) .
\end{aligned}
$$

And $\widetilde{\omega}_{j}^{n}$ should satisfy the following:

$$
\begin{aligned}
\widetilde{\omega}_{j}^{n}= & \frac{1}{2+\lambda} \widetilde{\omega}_{j-1}^{n}+\frac{1}{2+\lambda} \widetilde{\omega}_{j+1}^{n}+\left(\frac{\lambda}{2+\lambda}\right) \widetilde{\omega}_{j}^{n-1} \\
& -\frac{\lambda \Delta t}{2+\lambda} R(n \Delta t) .
\end{aligned}
$$

Substituting (22) and (23) into (21),

$$
\varepsilon_{j}^{n}=\frac{1}{2+\lambda} \varepsilon_{j-1}^{n}+\frac{1}{2+\lambda} \varepsilon_{j+1}^{n}+\left(\frac{\lambda}{2+\lambda}\right) \varepsilon_{j}^{n-1}+O(\Delta t) .
$$

Because the coefficients in (24) are positive,

$$
\begin{aligned}
\left|\varepsilon_{j}^{n}\right| \leq & \frac{1}{2+\lambda}\left|\varepsilon_{j-1}^{n}\right|+\frac{1}{2+\lambda}\left|\varepsilon_{j+1}^{n}\right|+\left(\frac{\lambda}{2+\lambda}\right)\left|\varepsilon_{j}^{n-1}\right| \\
& +\left|Z_{j}^{n-1}\right|
\end{aligned}
$$

where $Z_{j}^{n-1}=O(\Delta t)$.

It can be shown that

$$
\begin{aligned}
\varepsilon_{\max }^{n} \leq & \frac{1}{2+\lambda} \varepsilon_{\max }^{n-1}+\frac{1}{2+\lambda} \varepsilon_{\max }^{n-1}+\left(\frac{\lambda}{2+\lambda}\right) \varepsilon_{\max }^{n-1} \\
& +Z_{\text {max }}^{n-1},
\end{aligned}
$$

where

$$
\begin{array}{cc}
\varepsilon_{\max }^{n-1}=\max \left\{\left|\varepsilon_{j-1}^{n-1}\right|\right\} & 1 \leq j \leq N-1, \\
Z_{\max }^{n-1}=\max \left\{\left|Z_{j}^{n-1}\right|\right\} & 1 \leq j \leq N-1,
\end{array}
$$

and that

$$
\varepsilon_{\max }^{n} \leq \varepsilon_{\max }^{n-1}+Z_{\max }^{n-1} \quad(n=1,2, \ldots, M) .
$$

Because the finite analytical equation and the partial differential equation have the same initial condition, namely, $n=$ 1 , the finite analytical solution is consistent with the exact solution. That is, $\varepsilon_{\max }^{0}=0$.

From (27), the following expressions can be obtained:

$$
\begin{gathered}
\varepsilon_{\max }^{1} \leq \varepsilon_{\max }^{0}+Z_{\max }^{0}=Z_{\max }^{0}, \\
\varepsilon_{\max }^{2} \leq \varepsilon_{\max }^{1}+Z_{\max }^{1} \leq Z_{\max }^{0}+Z_{\max }^{1}
\end{gathered}
$$

$$
\varepsilon_{\max }^{M} \leq \varepsilon_{\max }^{M-1}+Z_{\max }^{M-1} \leq Z_{\max }^{0}+Z_{\max }^{1}+\cdots+Z_{\max }^{M-1} .
$$

Therefore,

$$
\varepsilon_{\max }^{M} \leq M \cdot Z_{\max }=M \cdot \max |O(\Delta t)|,
$$

where

$$
Z_{\max }=\max \left\{Z_{\max }^{n}\right\} \quad 1 \leq n \leq M .
$$


When $\Delta t \rightarrow 0, \varepsilon_{\max }^{M} \rightarrow 0$, thus $\widetilde{\omega}_{j}^{n} \rightarrow \omega_{j}^{n}$. Thus, it revealed that the finite analytical scheme has uniform convergence.

4.2. Stability of the Finite Analytical Numerical Scheme. The stability of finite analytical scheme (19) can be proven as follows. Let $\boldsymbol{\omega}^{n}=\left(\begin{array}{llll}\omega_{0}^{n} & \omega_{1}^{n} & \cdots & \omega_{m}^{n}\end{array}\right)^{T}$; taking account of calculation error, $\boldsymbol{\omega}^{0}$ becomes $\boldsymbol{\omega}^{0 *}$ and $\boldsymbol{\omega}^{n}$ becomes $\boldsymbol{\omega}^{n *}$. Thus, the error of any term is

$$
e_{j}^{n}=\omega_{j}^{n}-\omega_{j}^{n *}
$$

From (19), the error $e_{j}^{n}$ fits with

$$
-\frac{1}{\lambda} e_{j-1}^{n+1}+\frac{2+\lambda}{\lambda} e_{j}^{n+1}-\frac{1}{\lambda} e_{j+1}^{n+1}=e_{j}^{n}
$$

It can be proven that the error at $(n+1)$ th time step $e_{j}^{n+1}$ is between the error at the node 0 and the $(n+1)$ th time step $e_{0}^{n+1}$, the error at the node $m$ and the $(n+1)$ th time step $e_{m}^{n+1}$, and the extreme of the error in $n$th time step $e_{j}^{n}$; that is,

$$
m^{n} \leq e_{j}^{n+1} \leq M^{n}
$$

where

$$
\begin{aligned}
m^{n} & =\min \left\{e_{0}^{n+1}, e_{j}^{n}, e_{m}^{n+1}\right\}, \\
M^{n} & =\max \left\{e_{0}^{n+1}, e_{j}^{n}, e_{m}^{n+1}\right\} .
\end{aligned}
$$

Equation (34) can be proven as follows. Assume $e_{k}^{n+1}=$ $\min _{j} e_{j}^{n+1}$. When $k=0$ or $k=m$, there is $e_{j}^{n+1} \geq m^{n}$ clearly. When $0<k<m$, taking into account (33),

$$
\begin{aligned}
e_{k}^{n+1} & =e_{k}^{n}+\frac{1}{\lambda}\left(e_{k-1}^{n+1}+e_{k+1}^{n+1}-2 e_{k}^{n+1}\right) \\
& \geq e_{k}^{n}+\frac{1}{\lambda}\left(e_{k}^{n+1}+e_{k}^{n+1}-2 e_{k}^{n+1}\right)=e_{k}^{n} \geq m^{n} .
\end{aligned}
$$

Evidenced by the same token, $e_{k}^{n+1} \leq M^{n}$.

The finite analytical equation and the partial differential equation have the same boundary condition, namely, $e_{0}^{n}=$ $e_{m}^{n}=0$, and taking into account (34),

$$
\min _{j} e_{j}^{n} \leq e_{j}^{n+1} \leq \max _{j} e_{j}^{n}
$$

Therefore,

$$
\left\|\mathrm{e}^{n+1}\right\|_{\infty}=\max _{j}\left|e_{j}^{n+1}\right| \leq \max _{j}\left|e_{j}^{n}\right|=\left\|\mathbf{e}^{n}\right\|_{\infty} .
$$

The following expression can be obtained:

$$
\left\|\mathrm{e}^{n+1}\right\|_{\infty} \leq\left\|\mathrm{e}^{n}\right\|_{\infty} \leq \cdots \leq\left\|\mathrm{e}^{0}\right\|_{\infty} .
$$

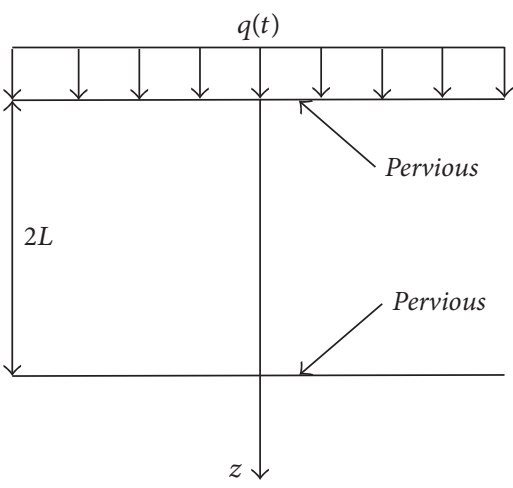

FIGURE 2: 1D foundation model.

Equation (39) reveals that the finite analytical scheme (19) is unconditionally stable.

\section{Semianalytical Solution of Governing Differential Equation}

The analytical model fitting with all assumptions except hypothesis (c) mentioned in Section 2 is shown in Figure 2. The depth of the soft clay with permeable top and bottom is $2 L$. Time-dependent loading $q(t)$ is imposed on top of the soft clay.

According to definition, in Figure 2, the initial and boundary conditions of the problem are

$$
\begin{gathered}
u(z, 0)=q(0) \quad \text { if } 0 \leq z \leq 2 L, t=0, \\
u(0, t)=0 \quad \text { if } z=0, t>0, \\
u(2 L, t)=0 \quad \text { if } z=2 L, t>0 .
\end{gathered}
$$

By using (6), the transform of (40) is

$$
\begin{gathered}
\omega(z, 0)=Q(0) \quad \text { if } 0 \leq z \leq 2 L, t=0, \\
\omega(0, t)=0 \quad \text { if } z=0, t>0, \\
\omega(2 L, t)=0 \quad \text { if } z=2 L, t>0,
\end{gathered}
$$

where

$$
Q(t)=\frac{\left(1+e_{0}\right) \sigma^{\prime}}{E+n \sigma^{\prime}}-\frac{\left(1+e_{0}\right) q(t)}{E+n q(t)} .
$$

When hypothesis (c) is not considered, the general solution of the problem is modified as

$$
u=\frac{\omega(z, t)[E+n q(t)]^{2}}{n \omega(z, t)[E+n q(t)]-E\left(1+e_{0}\right)}
$$


The average degrees of consolidation of the soil defined in pore-water pressure $U_{p}$ can be given by

$$
\begin{aligned}
U_{p} & =\frac{\int_{0}^{2 L}\left(\sigma^{\prime}-\sigma_{0}^{\prime}\right) d z}{\int_{0}^{2 L}\left(\sigma_{f}^{\prime}-\sigma_{0}^{\prime}\right) d z}=\frac{\int_{0}^{2 L}[q(t)-u] d z}{\int_{0}^{2 L} q_{u} d z} \\
= & \frac{q(t)}{q_{u}} \\
& -\frac{1}{2 L q_{u}} \int_{0}^{2 L} \frac{\omega(z, t)[E+n q(t)]^{2}}{n \omega(z, t)[E+n q(t)]-E\left(1+e_{0}\right)} d z,
\end{aligned}
$$

where

$$
\begin{aligned}
\omega(z, t)= & -\sum_{m=1}^{\infty} \frac{4}{m \pi} e^{-C_{v}(m \pi / 2 L)^{2} t} \sin \left(\frac{m \pi z}{2 L}\right) \\
& {\left[\int_{0}^{t} R(\tau) e^{C_{v}(m \pi / 2 L)^{2} \tau} d \tau-Q(0)\right] } \\
m & =1,3,5, \ldots .
\end{aligned}
$$

The average degrees of consolidation $U_{p}$ can be approximately written as follows:

$$
\begin{aligned}
U_{p} & =\frac{\int_{0}^{2 L}\left(\sigma^{\prime}-\sigma_{0}^{\prime}\right) d z}{\int_{0}^{2 L}\left(\sigma_{f}^{\prime}-\sigma_{0}^{\prime}\right) d z}=\frac{\int_{0}^{2 L}[q(t)-u] d z}{\int_{0}^{2 L} q_{u} d z} \\
& =\frac{q(t)-(1 / 2 L) \sum_{j=1}^{j=2 L / \Delta z} u_{j} \Delta z}{q_{u}} .
\end{aligned}
$$

According to (43) and (44), the exact expression of $\omega(z, t)$ is the key to obtaining excess pore-water pressure and average degrees of consolidation for different timedependent loadings. The following sections will discuss each of $\omega(z, t)$ under suddenly imposed constant loading, single ramp loading, and trapezoidal cyclic loading. (a) Suddenly Imposed Constant Loading

$$
q(t)=q_{u}
$$

Therefore,

$$
Q(0)=-\frac{\left(1+e_{0}\right) q_{u}}{E+n q_{u}} .
$$

Substituting (47) and (48) into (45),

$$
\begin{aligned}
\omega(z, t)= & \sum_{m=1}^{\infty} \frac{4}{m \pi} \sin \left(\frac{m \pi z}{2 L}\right) \\
& \cdot e^{-C_{v}(m \pi / 2 L)^{2} t} \frac{\left(1+e_{0}\right) q_{u}}{E+n q_{u}} \quad m=1,3,5, \ldots
\end{aligned}
$$

Substituting (49) into (43) and (44) or (46), the excess pore-water pressure and average degrees of consolidation can be obtained for suddenly imposed constant loading.

(b) Single Ramp Loading

$$
q(t)= \begin{cases}\frac{q_{u}}{t_{0}} t, & \text { if } t \leq t_{0} \\ q_{u}, & \text { if } t>t_{0}\end{cases}
$$

Therefore,

$$
Q(0)=0 \text {. }
$$

From (7) and (50),

$$
R(t)= \begin{cases}\frac{E\left(1+e_{0}\right) q_{u} / t_{0}}{\left[E+n\left(q_{u} / t_{0}\right) t\right]^{2}}, & t \leq t_{0}, \\ 0, & t>t_{0} .\end{cases}
$$

The semianalytical solution to the problem is

$$
\begin{aligned}
& \omega(z, t) \\
& =\left\{\begin{array}{l}
-\sum_{m=1}^{\infty} \frac{4}{m \pi} \sin \left(\frac{m \pi z}{2 L}\right)\left(\left(E i\left(1,-\frac{b d}{c}\right)-E i\left(1,-\frac{d(t c+b)}{c}\right)\right) \cdot\left(t b c d+b^{2} d\right)-e^{d(t c+b) / c} b c+e^{b d / c}\left(t c^{2}+b c\right)\right) \frac{a e^{-d(t c+b) / c}}{b c^{2}(t c+b)}, \quad \text { if } t \leq t_{0}, \\
-\sum_{m=1}^{\infty} \frac{4}{m \pi} \sin \left(\frac{m \pi z}{2 L}\right)\left(\left(E i\left(1,-\frac{b d}{c}\right)-E i\left(1,-\frac{d\left(t_{0} c+b\right)}{c}\right)\right) \cdot\left(t_{0} b c d+b^{2} d\right)-e^{d\left(t_{0} c+b\right) / c} b c+e^{b d / c}\left(t_{0} c^{2}+b c\right)\right) \frac{a e^{-d\left(\left(t+t_{0}\right) c+b\right) / c}}{b c^{2}\left(t_{0} c+b\right)}, \quad \text { if } t>t_{0},
\end{array}\right. \\
& m=1,3,5, \ldots,
\end{aligned}
$$

where

$$
\begin{aligned}
d & =C_{v}\left(\frac{m \pi}{2 L}\right)^{2}, \\
\operatorname{Ei}\left(a_{1}, b_{1}\right) & =\int_{1}^{\infty} e^{-x b_{1}} x^{-a_{1}} d x,
\end{aligned}
$$$$
a=\frac{E\left(1+e_{0}\right) q_{u}}{t_{0}},
$$

$b=E$,

$c=n \frac{q u}{t_{0}}$ $a_{1}$ and $b_{1}$ are nonzero constants.

Substituting (53) into (43) and (44) or (46), the excess pore-water pressure and average degrees of consolidation can be obtained for single ramp loading. 
(c) Trapezoidal Cyclic Loading (Figure 3)

$$
q(t)= \begin{cases}\frac{q_{u}}{\alpha t_{0}}\left[t-(N-1) \beta t_{0}\right], & \text { if }(N-1) \beta t_{0} \leq t \leq[(N-1) \beta+\alpha] t_{0}, \\ q_{u}, & \text { if }[(N-1) \beta+\alpha] t_{0} \leq t \leq[(N-1) \beta+(1-\alpha)] t_{0}, \\ -\frac{q_{u}}{\alpha t_{0}}\left[t-(N-1) \beta t_{0}-t_{0}\right], & \text { if }[(N-1) \beta+(1-\alpha)] t_{0} \leq t \leq[(N-1) \beta+1] t_{0}, \\ 0, & \text { if }[(N-1) \beta+1] t_{0} \leq t \leq N \beta t_{0},\end{cases}
$$

where $q_{u}$ is the maximum value of loading during a cycle period; $\beta t_{0}$ is the period of the loading; $\alpha, \beta$ are loading factors; $N$ is the cycle number.

$$
R(t)=\left\{\begin{array}{l}
\frac{E\left(1+e_{0}\right) q_{u} /\left(\alpha t_{0}\right)}{\left[E+n\left(q_{u} / \alpha t_{0}\right)\left(t-(N-1) \beta t_{0}\right)\right]^{2}} \\
0, \\
-\frac{E\left(1+e_{0}\right) q_{u} /\left(\alpha t_{0}\right)}{\left[E-n\left(q_{u} / \alpha t_{0}\right)\left(t-((N-1) \beta+1) t_{0}\right)\right]^{2}} \\
0,
\end{array}\right.
$$

The semianalytical solution for consolidation under the loading case is shown in the Appendix. It is clear that the form of the solution is complex. Compared with the semianalytical solution (A.1), the general solution, obtained by using (45) and (57), can be easier to program.

\section{Comparison between Results from FAM and Semianalytical Solutions}

The numerical solution of FAM is compared with the semianalytical solution for different loading cases. The value of constant parameters from [26] are $C_{v}=5.789 \times 10^{-3} \mathrm{~m}^{2} /$ day, $E=1.6878 \times 10^{6} \mathrm{~Pa}, n=3.3, e_{0}=1.1167,2 L=6.4 \mathrm{~m}$, and $t=800$ days. In the FAM, the time step $\Delta t$ is 0.1 days.

(a) Suddenly Imposed Constant Loading Case. For the suddenly imposed constant loading case, $q_{u}=2 \times 10^{5} \mathrm{~Pa}$. The duration of loading is 800 days. The average degrees of consolidation $U_{p}$ is calculated by using (46), adopting the same space step $\Delta z$ for FAM and the semianalytical solution. $\omega(z, t)$ is calculated by using (49). The number of terms in the series is 20 . Curves of the average degrees of consolidation $U_{p}$ versus time factor $T_{v}$ (shown in (58)) for different methods with different space steps $\Delta z$ are plotted in Figure 4 (ASM indicates "semianalytical solution method" in the figure). Comparison between the result of FAM and the semianalytical solution shows a negligible gap, indicating that the method proposed in this paper has sufficient precision.

$$
T_{v}=C_{v} t L^{-2}
$$

Therefore,

$$
Q(0)=0 \text {. }
$$

From (7) and (50),

$$
\begin{aligned}
& \text { if }(N-1) \beta t_{0} \leq t \leq[(N-1) \beta+\alpha] t_{0}, \\
& \text { if }[(N-1) \beta+\alpha] t_{0} \leq t \leq[(N-1) \beta+(1-\alpha)] t_{0}, \\
& \text { if }[(N-1) \beta+(1-\alpha)] t_{0} \leq t \leq[(N-1) \beta+1] t_{0}, \\
& \text { if }[(N-1) \beta+1] t_{0} \leq t \leq N \beta t_{0} .
\end{aligned}
$$

In order to investigate the accuracy of this result further, the percentage deviation of $U_{p}$ between FAM and semianalytical solutions is evaluated. To illustrate the difference of $U_{p}$ between the two, 21 data points of the percentage deviation of $U_{p}$ are chosen at regular intervals for each data group plotted in Figure 5. The percentage deviation decreases with decreasing space step $\Delta z$, demonstrating that, under the same conditions, a smaller space step is helpful in enhancing the precision of calculation. However, there is a clear side effect: the amount of calculation increases. Considering the precision and the amount of calculation, $\Delta z=0.2 \mathrm{~m}$ is selected in the next two loading cases: single ramp loading case and trapezoidal cyclic loading case.

(b) Single Ramp Loading Case. In the single ramp loading case, $q_{u}=2 \times 10^{5} \mathrm{~Pa}$. The value of $q(t)$ increases linearly from 0 to $q_{u}$ during the loading increase phase $t_{0}$. In this section, the influence of the loading increase (phase $t_{0}$ ) is discussed. The loading increasing (phase $t_{0}$ ) is $141.5,283$, and 424.5 days, respectively, and the value of $q(t)$ remains constant. Further $\omega(z, t)$ is calculated by using (53) in the semianalytical solution. In order to avoid overflow, the number of terms in the series is 6 . The curves of the average degrees of consolidation $U_{p}$ versus time factor $T_{v}$ for different methods with different loading increase periods are plotted in Figure 6. As shown in Figure 6, $U_{p}$ increases with increasing $T_{v}$, but there is a turning point after which the rate of increase slows down. For the different loading increase (period $t_{0}$ ), the degree of consolidation with a short loading increase is higher than the degree of consolidation with a long loading increase period. 


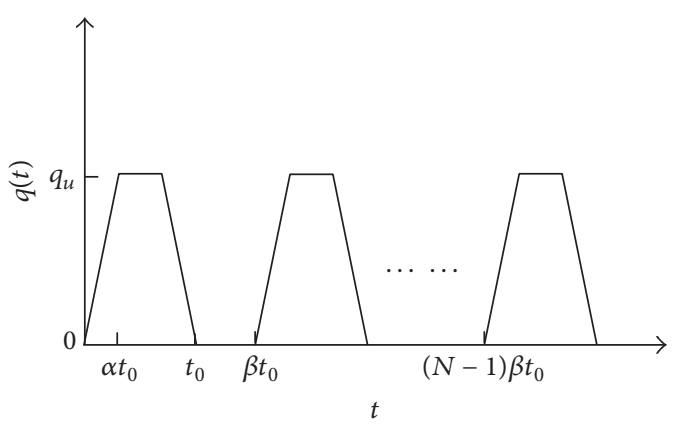

Figure 3: Trapezoidal cyclic loading.

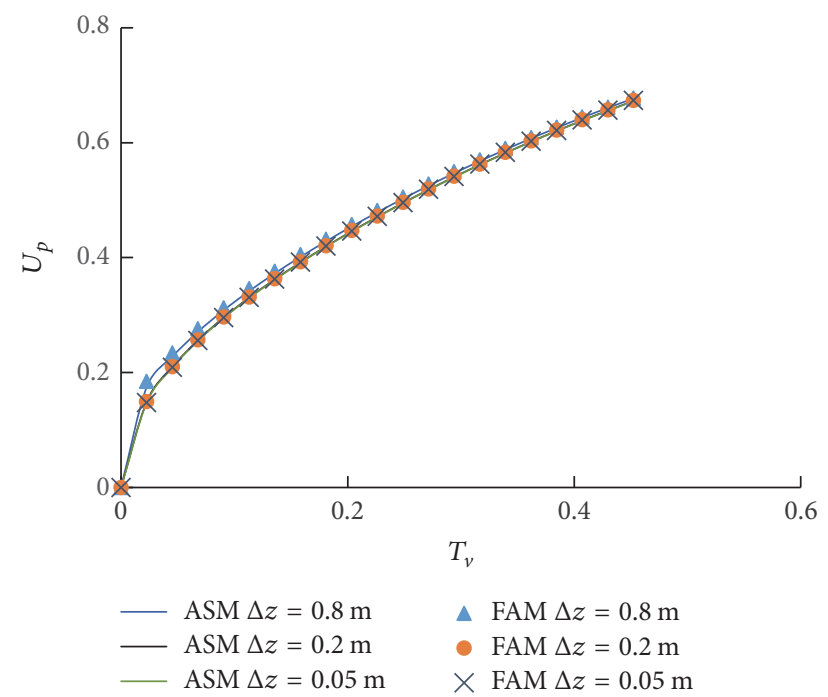

Figure $4: U_{p}$ versus $T_{v}$ curves for constant loading.

Figure 6 also shows that the agreement between the curves obtained by using the FAM and the semianalytical solutions is quite remarkable. To illustrate the difference of $U_{p}$ between the FAM and the semianalytical solution under single ramp loading case, 21 data points of the percentage deviation of $U_{p}$ are chosen at regular intervals for each data group plotted in Figure 7. Figure 7 shows that the percentage deviation of $U_{p}$ between the FAM and the semianalytical solution changes with increasing $T_{v}$. Although there are differences in different loading increase period cases, the percentage deviation of $U_{p}$ is generally negligible.

(c) Trapezoidal Cyclic Loading Case. In the trapezoidal cyclic loading case, the maximum value of loading $q_{u}$ during a cycle period is $2 \times 10^{5} \mathrm{~Pa}$. The period of the loading is written as $\beta t_{0}$. $t_{0}$ is a loading period of 141.5 days. The loading factors $\alpha$ and $\beta$ are 0.3 and 1.0, 0.3 and 1.2, 0.3 and 2.4, and 0.5 and 1.2, which represent trapezoidal cyclic loading without a rest period, trapezoidal cyclic loading with $0.2 t_{0}$ rest period, trapezoidal cyclic loading with $1.4 t_{0}$ rest period, and triangularly cyclic loading with a $0.2 t_{0}$ rest period, respectively. Here, $\omega(z, t)$ is calculated by using (45) in the semianalytical solution. In order to avoid overflow, the number of terms in the series

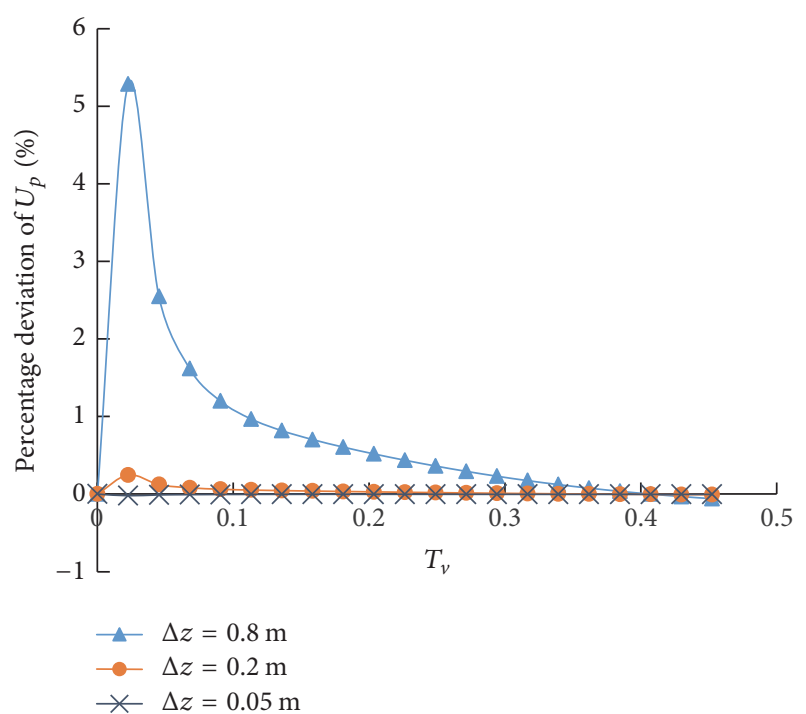

FIgURe 5: Percentage deviation of $U_{p}$ between the FAM and the semianalytical solution versus $T_{v}$ curves for constant loading.

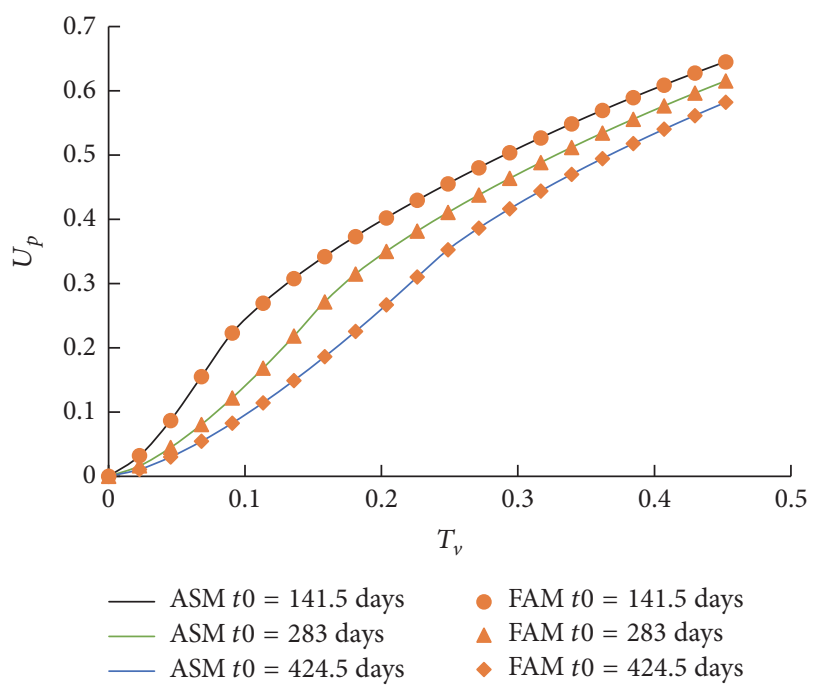

FIgURE 6: $U_{p}$ versus $T_{v}$ curves for single ramp loading.

is 10. The curves of the average degrees of consolidation $U_{p}$ versus time factor $T_{v}$ with different loading factors $\alpha$ and $\beta$ for different methods are plotted in Figure 8, showing that $U_{p}$ tends to be fluctuating while increasing with increasing $T_{v}$. To illustrate the difference of $U_{p}$ between the FAM and the semianalytical solution under trapezoidal cyclic loading case, 21 data points of the percentage deviation of $U_{p}$ are chosen at regular intervals for each data group plotted in Figure 8. The comparison between the result of FAM and the semianalytical solution shows a negligible gap in Figure 8. The percentage deviation of $U_{p}$ between the FAM and the semianalytical solution for trapezoidal cyclic loading is plotted in Figure 9, indicating that fluctuation deviation is small in range in terms of percent and is, thus, negligible. 


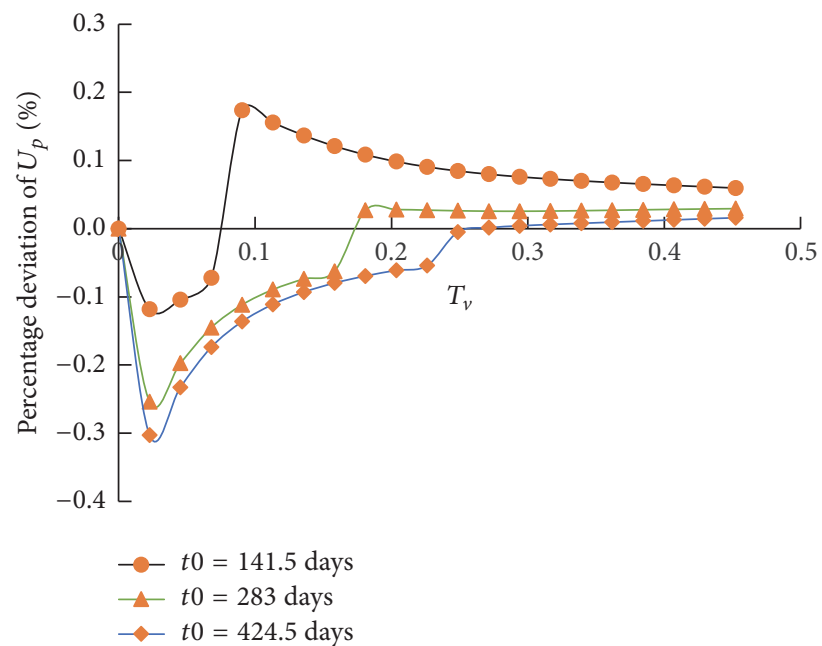

Figure 7: Percentage deviation of $U_{p}$ between the FAM and the semianalytical solution versus $T_{v}$ curves for single ramp loading.

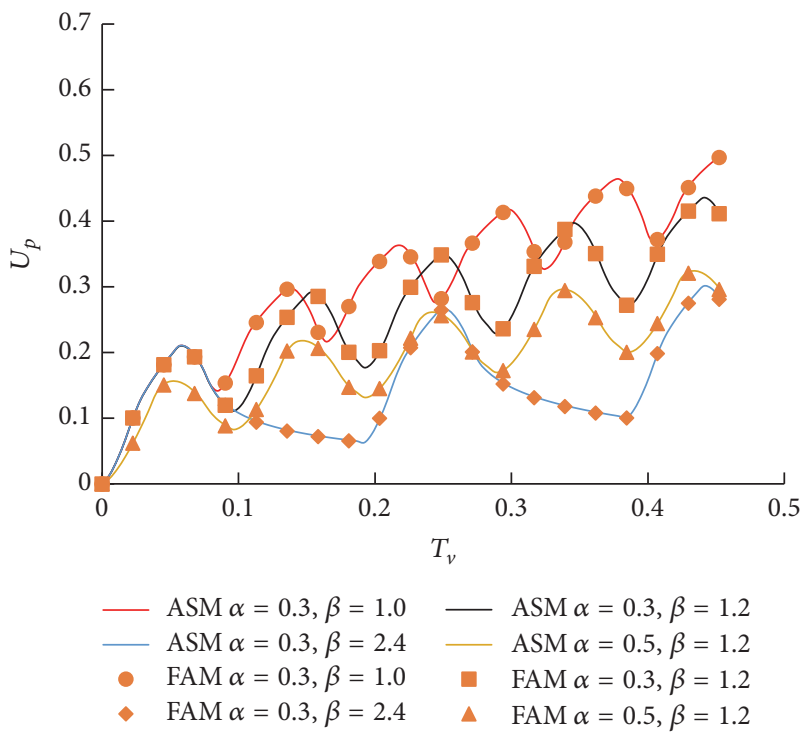

FIGURE 8: $U_{p}$ versus $T_{v}$ curves for trapezoidal cyclic loading.

\section{Convergence of the Spatial Discretization for FAM}

Proving the convergence of the spatial discretization by a mathematical approach is difficult. To further explore the convergence of the spatial discretization, a $1 \mathrm{D}$ nonlinear consolidation in the trapezoidal cyclic loading case was introduced as an example. The initial and boundary conditions are as follows:

$$
\begin{gathered}
u(z, 0)=10^{4} z, \quad \text { if } 0 \leq z \leq L, t=0, \\
u(0, t)=0, \quad \text { if } z=0, t>0, \\
\frac{\partial u(L, t)}{\partial z}=0, \quad \text { if } z=L, t>0 .
\end{gathered}
$$

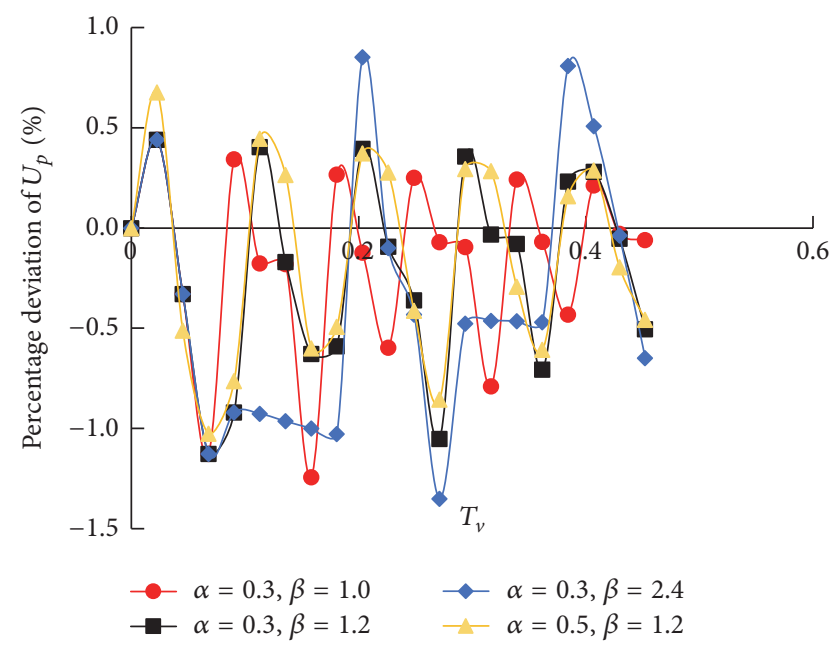

Figure 9: Percentage deviation of $U_{p}$ between the FAM and the semianalytical solution versus $T_{v}$ curves for trapezoidal cyclic loading.

By using (6), the transform of (59) is

$$
\begin{aligned}
\omega(z, 0) & =\frac{-2.1167 z}{168.78-3.3 z}, \quad \text { if } 0 \leq z \leq L, t=0, \\
\omega(0, t) & =0, \quad \text { if } z=0, t>0, \\
\frac{\partial \omega(L, t)}{\partial t} & =0, \quad \text { if } z=L, t>0 .
\end{aligned}
$$

In this example, a soft clay layer with permeable top and impermeable bottom under initial excess pore-water pressure triangular distribution is introduced, where $q_{u}=2 \times 10^{5} \mathrm{~Pa}$, $t_{0}=141.5$ days, $\alpha=0.3$, and $\beta=1.2$. The values of the constant parameters are the same as those in Section 6. However, for the case, the analytical solution is difficult to be obtained. But it is easy to solve by numerical method, such as FAM and FDM. For comparison, the space step $\Delta z$ is set to $0.1,0.2,0.4$, and $0.8 \mathrm{~m}$ by FAM, respectively. To achieve good solutions as comparison, the space step $\Delta z$ is adopted to $0.1 \mathrm{~m}$ by FDM. The space step has influence on degree of consolidation calculated value of $U_{p}$. To avoid this, the excess pore-water pressure $u$ calculated by FAM with different space steps is compared with FDM with specific space step $\Delta z=0.1 \mathrm{~m}$, shown in Figure 10. It reveals that the results of FAM matches well with the result of FDM, even if $\Delta z$ is $0.8 \mathrm{~m}$. Therefore, FAM can ensure the convergence of spatial discretization.

\section{Conclusions}

This paper develops the finite analytic method (FAM) for solving a 1D nonlinear consolidation undergoing timedependent loading. The study shows that FAM is very efficient and convenient in simulating the nonlinear consolidation. The following conclusions can be drawn from this research:

(1) The governing partial differential equation with nonhomogeneous character can be transformed into the 

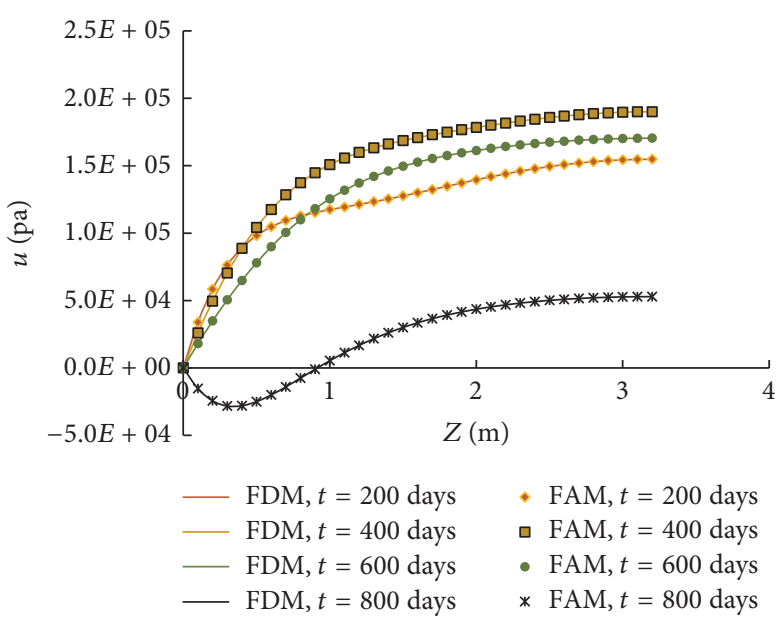

(a) $\operatorname{FDM} \Delta z=0.1 \mathrm{~m}$; FAM $\Delta z=0.1 \mathrm{~m}$

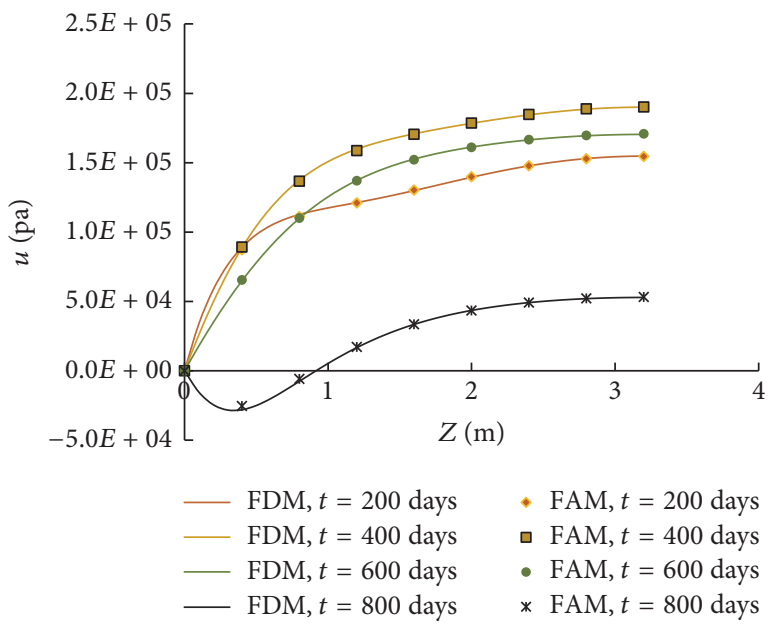

(c) FDM $\Delta z=0.1 \mathrm{~m}$; FAM $\Delta z=0.4 \mathrm{~m}$

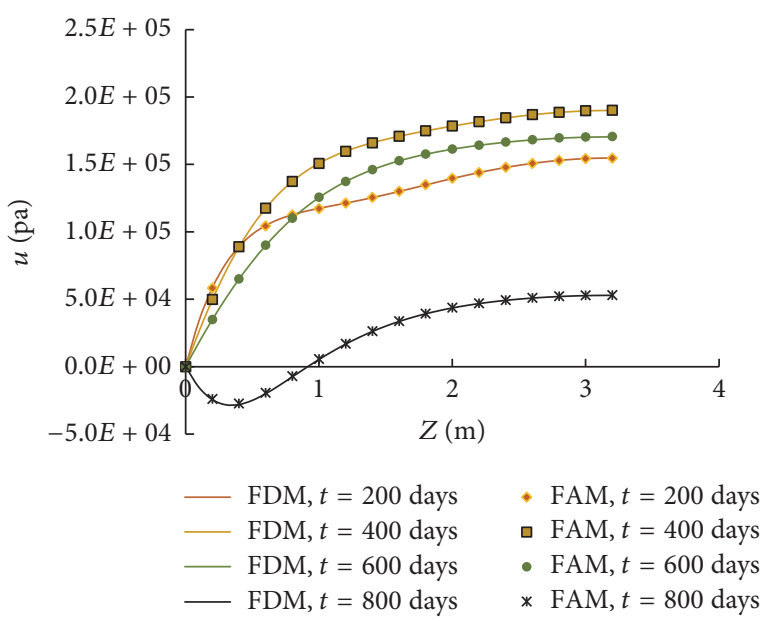

(b) FDM $\Delta z=0.1 \mathrm{~m}$; FAM $\Delta z=0.2 \mathrm{~m}$

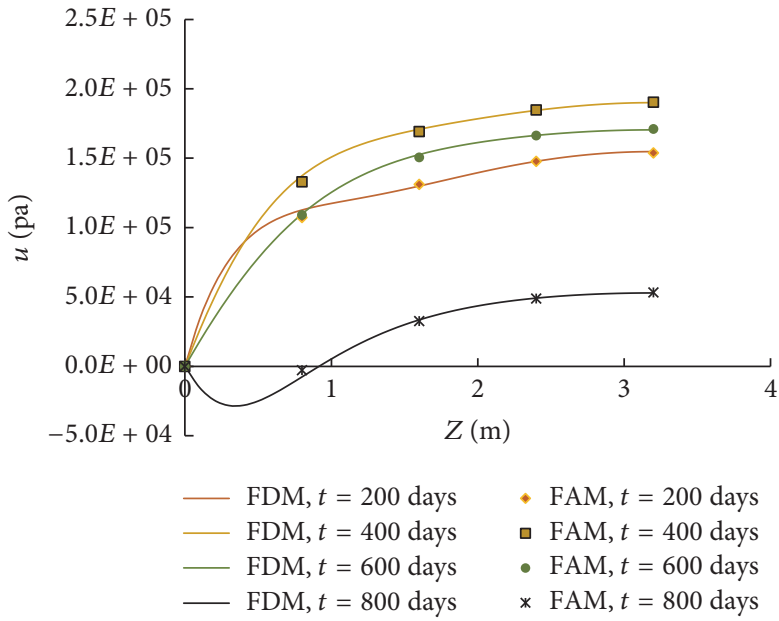

(d) FDM $\Delta z=0.1 \mathrm{~m}$; FAM $\Delta z=0.8 \mathrm{~m}$

FIgURE 10: Comparison between the results of FAM and the results of FDM.

diffusion equation, where the FAM uses an analytical solution in small local elements to formulate the algebraic representation of the $1 \mathrm{D}$ nonlinear consolidation undergoing time-dependent loading.

(2) The FAM is a combination of analytical solution and numerical approach. The finite analytical scheme has uniform convergence and is unconditionally stable for 1D nonlinear consolidation. Therefore, FAM can obtain smooth and high-precision solutions.

(3) The comparison between the results of FAM and the semianalytical solution of the $1 \mathrm{D}$ nonlinear consolidation undergoing suddenly imposed constant loading, single ramp loading, and trapezoidal cyclic loading indicates that FAM can obtain stable and accurate numerical solutions.

(4) The comparison between the results of FAM with different space steps and the results of FDM with a small space step indicates that FAM can ensure the convergence of spatial discretization.

\section{Appendix}

The modified semianalytical solution of $\omega(z, t)$ of a trapezoidal cyclic loading case is as follows:

$$
\begin{aligned}
\omega(z, t)= & -\sum_{m=1}^{\infty} \frac{4}{m \pi} e^{-C_{v}(m \pi / 2 L)^{2} t} \\
& \cdot \sin \left(\frac{m \pi z}{2 L}\right)\left[T_{m N}+T_{m i}\right],
\end{aligned}
$$

where $m=1,3,5, \ldots ; i=1,2,3,4$ represents time $t$ lying in different loading phase during a loading cycle. $T_{m N}$ represents the integration for variable $\tau$ from 0 to $(N-1) \beta t_{0}$ :

$$
\begin{aligned}
T_{m N} & =\int_{0}^{(N-1) \beta t_{0}} R(\tau) e^{C_{v}(m \pi / 2 L)^{2} \tau} d \tau \\
& =\sum_{k=1}^{N-1}\left\{\int_{(k-1) \beta t_{0}}^{[(k-1) \beta+\alpha] t_{0}} \frac{E\left(1+e_{0}\right)\left(q_{u} / \alpha t_{0}\right) e^{C_{v}(m \pi / 2 L)^{2} \tau}}{\left[E+n q_{u}\left[\tau-(k-1) \beta t_{0}\right] / \alpha t_{0}\right]^{2}} d \tau\right. \\
& \left.-\int_{[(k-1) \beta+(1-\alpha)] t_{0}}^{[(k-1) \beta+1] t_{0}} \frac{E\left(1+e_{0}\right)\left(q_{u} / \alpha t_{0}\right) e^{C_{v}(m \pi / 2 L)^{2} \tau}}{\left[E-n q_{u}\left[\tau-(k-1) \beta t_{0}-t_{0}\right] / \alpha t_{0}\right]^{2}} d \tau\right\} .
\end{aligned}
$$


When $(N-1) \beta t_{0} \leq t \leq[(N-1) \beta+\alpha] t_{0}$ (Phase 1 ),

$T_{m 1}(t)$

$$
=\int_{(k-1) \beta t_{0}}^{t} \frac{E\left(1+e_{0}\right)\left(q_{u} / \alpha t_{0}\right) e^{C_{v}(m \pi / 2 L)^{2} \tau}}{\left[E+n q_{u}\left[\tau-(k-1) \beta t_{0}\right] / \alpha t_{0}\right]^{2}} d \tau .
$$

2),

When $[(N-1) \beta+\alpha] t_{0} \leq t \leq[(N-1) \beta+(1-\alpha)] t_{0}$ (Phase

$$
T_{m 2}(t)=T_{m 1}\left([(N-1) \beta+\alpha] t_{0}\right) .
$$

$3)$,

When $[(N-1) \beta+(1-\alpha)] t_{0} \leq t \leq[(N-1) \beta+1] t_{0}$ (Phase

$$
\begin{aligned}
& T_{m 3}(t)=T_{m 1}\left([(N-1) \beta+\alpha] t_{0}\right) \\
& \quad-\int_{[(N-1) \beta+(1-\alpha)] t_{0}}^{\tau} \frac{E\left(1+e_{0}\right)\left(q_{u} / \alpha t_{0}\right) e^{C_{\nu}(m \pi / 2 L)^{2} \tau}}{\left[E-n q_{u}\left[\tau-(k-1) \beta t_{0}-t_{0}\right] / \alpha t_{0}\right]^{2}} d \tau .
\end{aligned}
$$

When $[(N-1) \beta+1] t_{0} \leq t \leq N \beta t_{0}$ (Phase 4$)$,

$$
T_{m 4}(t)=T_{m 3}\left([(N-1) \beta+1] t_{0}\right) .
$$

\section{Conflicts of Interest}

The authors declare that there are no conflicts of interest regarding the publication of this paper.

\section{Acknowledgments}

This study was supported by National Natural Science Foundation of China (nos. 41230314, 41602237) and the Fundamental Research Funds for the Central Universities of China (nos. 310829151076, 310829161011, and 310829163307).

\section{References}

[1] D.-Y. Xie, Soil Dynamics, Xi'an Jiaotong University Press, Xian, China, 1987 (Chinese).

[2] J.-B. Xu, C.-G. Yan, X. Zhao, K. Du, H. Li, and Y.-L. Xie, "Monitoring of train-induced vibrations on rock slopes," International Journal of Distributed Sensor Networks, vol. 13, no. 1, pp. 1-7, 2017.

[3] J.-B. Xu, C.-G. Yan, H. Yuan et al., "The blasting vibration of deep buried soft rock tunnel," Electronic Journal of Geotechnical Engineering, vol. 22, no. 4, pp. 1219-1237, 2017.

[4] L.-J. Dong, J. Wesseloo, Y. Potvin, and X.-B. Li, "Discriminant models of blasts and seismic events in mine seismology," International Journal of Rock Mechanics and Mining Sciences, vol. 86, pp. 282-291, 2016.

[5] L.-J. Dong, X.-B. Li, and G.-N. Xie, "Nonlinear methodologies for identifying seismic event and nuclear explosion using random forest, support vector machine, and naive bayes classification," Abstract and Applied Analysis, vol. 2014, Article ID 459137, 8 pages, 2014.

[6] O. C. Zienkiewicz and P. Bettess, "Soils and other saturated media under transient, dynamic conditions," in Soil Mechanics-Transient and Cyclic Loads, pp. 1-16, John Wiley \& Sons, 1982.
[7] X. Y. Geng, C. J. Xu, and Y. Q. Cai, "Non-linear consolidation analysis of soil with variable compressibility and permeability under cyclic loadings," International Journal for Numerical and Analytical Methods in Geomechanics, vol. 30, no. 8, pp. 803-821, 2006.

[8] D. B. McLean, Permanent Deformation Characteristics of Asphalt Concrete, University of California, Berkeley, Calif, USA, 1974.

[9] Y. H. Huang, Pavement Analysis and Design, Prentice Hall, Englewood Cliffs, NJ, USA, 1993.

[10] K.-H. Xie, T. Qi, and Y.-Q. Dong, "Nonlinear analytical solution for one-dimensional consolidation of soft soil under cyclic loading," Journal of Zhejiang University: Science, vol. 7, no. 8, pp. 1358-1364, 2006.

[11] K. Terzaghi and O. K. Fröhlich, Theorie der Setzung von Tonschichten, Franz Deutike, Leipzig, Germany, 1936.

[12] R. E. Olson, "Consolidation under time-dependent loading," Journal of the Geotechnical Engineering Division, vol. 103, no. 1, pp. 55-60, 1977.

[13] M. Favaretti and M. Soranzo, "A simplified consolidation theory in cyclic loading conditions," in Proceedings of the International Symposium on Compression and Consolidation of Clayey Soils, pp. 405-409, A. A. Balkema, Hiroshima, Japan, May 1995.

[14] E. H. Davis and G. P. Raymond, "A non-linear theory of consolidation," Géotechnique, vol. 15, no. 2, pp. 161-173, 1965.

[15] S. S. Razouki, P. Bonnier, M. Datcheva, and T. Schanz, "Analytical solution for $1 \mathrm{D}$ consolidation under haversine cyclic loading," International Journal for Numerical and Analytical Methods in Geomechanics, vol. 37, no. 14, pp. 2367-2372, 2013.

[16] Y.-R. Zheng and L. Kong, Fundamentals of Geotechnical Plastic Mechanics, China Building Industry Press, Beijing, China, 1989 (Chinese).

[17] S.-M. Xu, "The normalization curve method for saturated clay foundation settlement calculation," Yan Tu Gong Cheng Xue Bao, vol. 9, no. 4, pp. 70-77, 1987 (Chinese).

[18] J.-Y. Shi, L.-A. Yang, and W.-B. Zhao, "Research of onedimensional consolidation theory considering nonlinear characteristics of soil," Journal of Hohai University, vol. 29, no. 1, pp. 1-5, 2001 (Chinese)

[19] L. Zhang, S.-L. Sun, X.-N. Gong, and J. Zhang, "Solution for one-dimensional consolidation based on hyperbola model under cyclic loading," Rock and Soil Mechanics, vol. 31, no. 2, pp. 456-460, 2010 (Chinese).

[20] C. J. Chen and P. Li, "The finite analytic method for steady and unsteady heat transfer problems," in Proceedings of the Joint National Heat Transfer Conference, pp. 80-86, American Society of Mechanical Engineers and American Institute of Chemical Engineers, Orlando, Fla, USA, July 1980.

[21] C.-J. Chen and H.-C. Chen, "Finite analytic numerical method for unsteady two-dimensional Navier-Stokes equations," Journal of Computational Physics, vol. 53, no. 2, pp. 209-226, 1984.

[22] W.-F. Tsai, C.-J. Chen, and H.-C. Tien, "Finite analytic numerical solutions for unsaturated flow with irregular boundaries," Journal of Hydraulic Engineering, vol. 119, no. 11, pp. 1274-1298, 1993.

[23] W.-K. Wang, Z.-X. Dai, J.-T. Li, and L.-L. Zhou, "A hybrid Laplace transform finite analytic method for solving transport problems with large Peclet and Courant numbers," Computers and Geosciences, vol. 49, pp. 182-189, 2012.

[24] Z.-Y. Zhang, W.-K. Wang, L. Chen et al., "Finite analytic method for solving the unsaturated flow equation," Vadose Zone Journal, vol. 14, no. 1, 2015. 
[25] Z.-Y. Zhang, W.-K. Wang, T.-C. J. Yeh et al., "Finite analytic method based on mixed-form Richards' equation for simulating water flow in vadose zone," Journal of Hydrology, vol. 537, pp. 146-156, 2016.

[26] L. Zhang, One-Dimensional Consolidation Theory Modified by Hyperbola Model under Generalized Time-Depending Loading, Hohai University, Nanjing, China, 2007 (Chinese). 


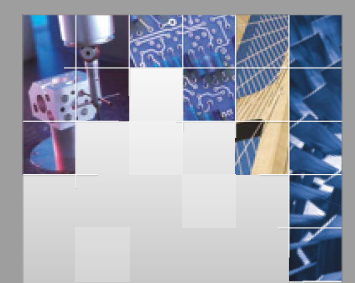

\section{Enfincering}
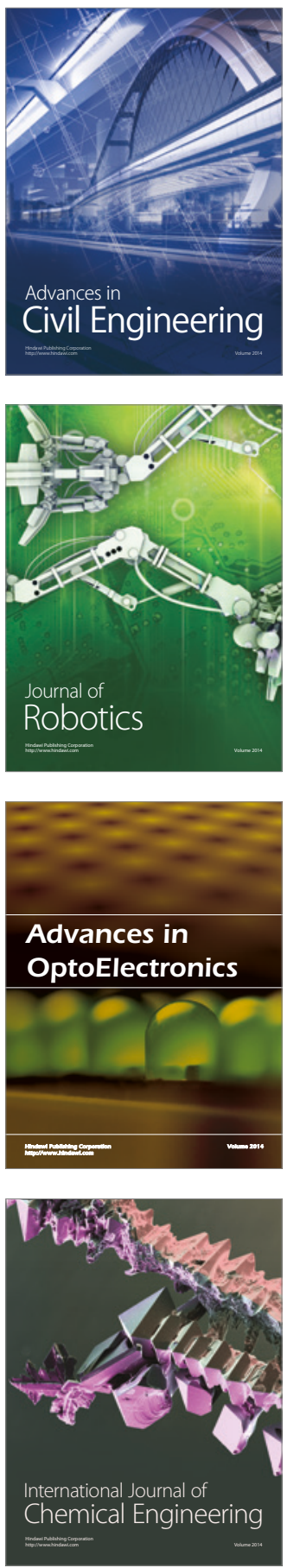

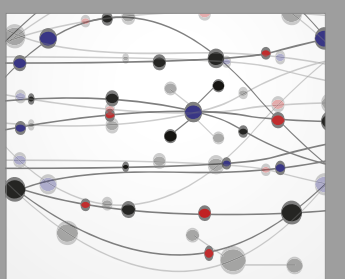

The Scientific World Journal

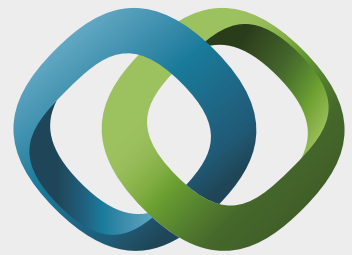

\section{Hindawi}

Submit your manuscripts at

https://www.hindawi.com
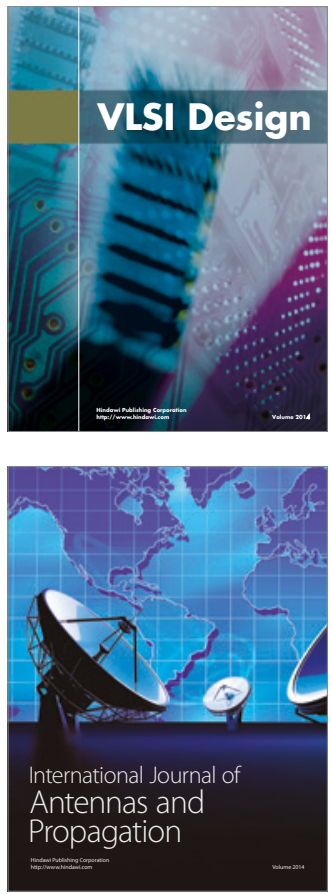

\section{Rotating}

Machinery
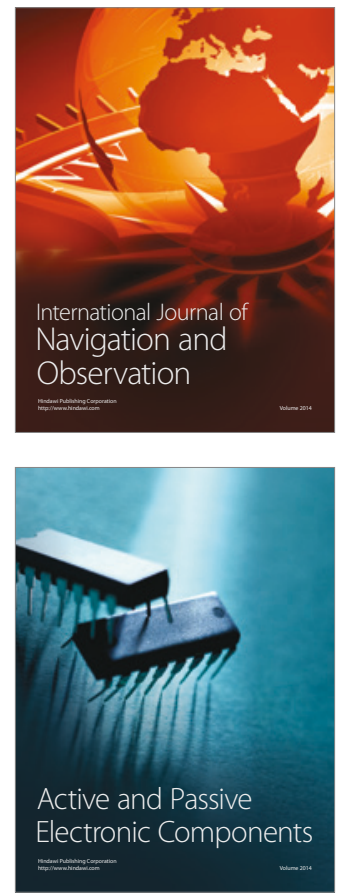
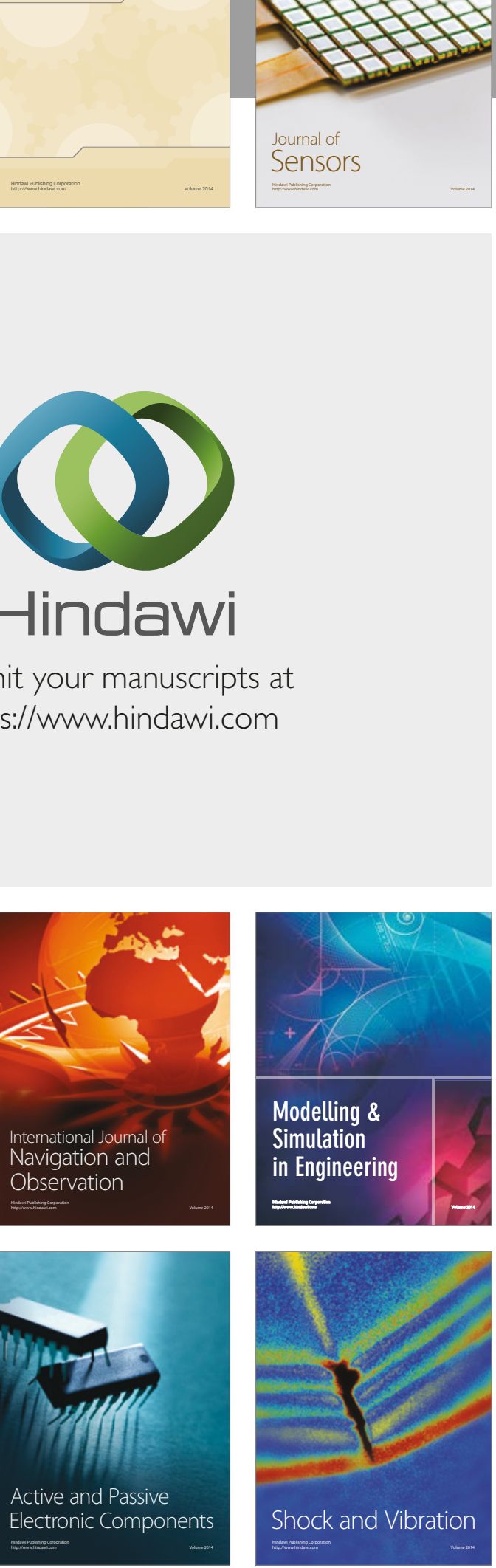
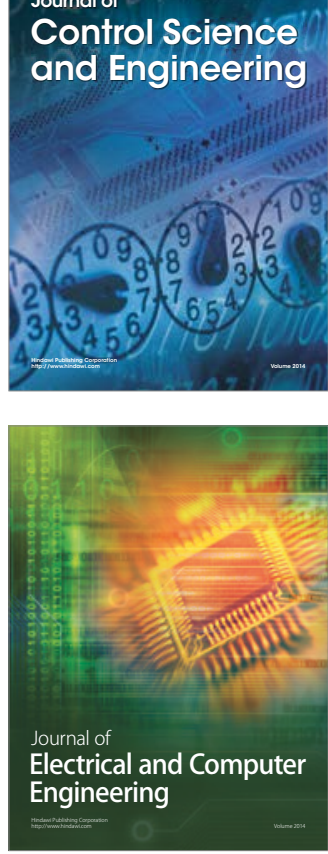

Distributed

Journal of

Control Science

and Engineering
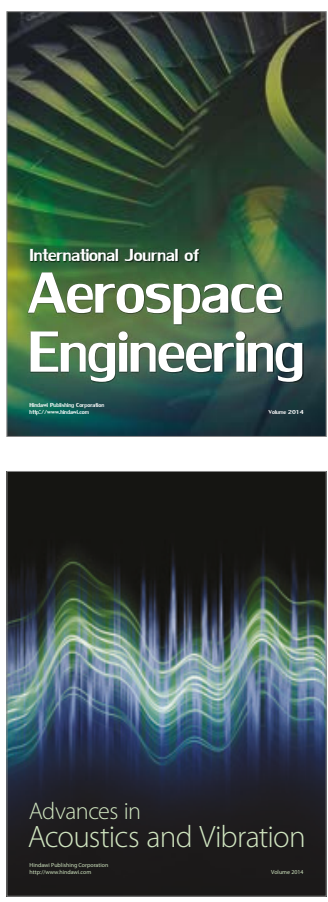

Sensor Networks 